\title{
Dynamic Photometric Stereo
}

\author{
Melvyn Smith and Lyndon Smith \\ Machine Vision Laboratory, \\ Faculty of Computing, Engineering and Mathematical Sciences (CEMS), \\ University of the West of England, Bristol, BS16 1QY, UK \\ Telephone 01173283578 \\ Melvyn.Smith@uwe.ac.uk \\ http: / /www. uwe.ac.uk/cems/research/melsmith/
}

\begin{abstract}
A new dynamic photometric stereo technique is presented, which facilitates the analysis of rapidly moving surfaces containing mixed two- and threedimensional concomitant features. A new approach termed narrow infra-red photometric stereo (NIRPS) is described as an evolution of the existing photometric stereo (PS) technique. The method has application for the inspection of web materials and other moving surfaces considered difficult to analyse using conventional imaging techniques. Experimental results are presented in the paper.
\end{abstract}

\section{Introduction}

Photometric stereo (PS) can be used to recover both local surface orientation and albedo using three, or more, separate images acquired under differing directional illumination conditions, where the surface reflectance is normally assumed to be Lambertian and the lighting and viewing positions are distant. Previous work [1-4] has shown how PS can be used in surface topographic analysis through the recovery and analysis of a dense array of surface normals in the form of a surface bump map. Applications range from texture analysis to surface inspection [1]. In order to maintain correspondence between successive images it is necessary that the scene remain static between image acquisitions, hence the images are separated in time, where each image is acquired one after the other. This form of temporally multiplexed works well in static applications using an area array camera hence we may term this approach static PS (SPS). However in numerous industrial inspection applications it is often the case that the surfaces to be inspected tend to be moving and often moving very rapidly, i.e. $>200 \mathrm{~m} /$ minute. Under such circumstances, conventional image acquisition is normally performed using linescan camera technology. It would be advantageous to be able to bring the benefits of PS to bear in on-line inspection of moving surfaces. However, achieving correspondence now becomes more problematic, as it may be impractical to capture three images. An alternative form of dynamic PS (DPS) is therefore proposed.

\section{Conventional Static Photometric Stereo}

The photometric stereo technique involves the capture of a number of views from a fixed spatial location while under differing carefully structured lighting configura- 
tions. By determining the normal at each pixel location, PS enables a full bump map to be obtained, which fully describes the surface topography. Smith [1, 4] has shown how the concept of a surface 'bump map', a term borrowed from computer graphics, could be used to represent a photometrically captured detailed 3D surface texture. In addition, it has also been shown how the bump map description offers opportunity for the characterisation of surface topographic features and textures in isolation from albedo through firstly mapping of the vector based data to a gradient space domain and then characterising the observed distribution [4]. Hence, by using what will be described here, as conventional, static or classical PS, precisely registered pixel based 2D albedo patterns and 3D surface topographic features and textures may be isolated and subsequently separately analysed [3]. A detailed explanation of PS will not be given here; instead the interested reader is referred to [1].

\subsection{The Problem of Acquiring Multiple Images of Moving Surfaces}

A limiting assumption forming the basis of SPS is that differing separate directional illumination configurations must be present in each of the acquired views of precisely the same region of the surface. While this is readily achieved in the static scenario, either by moving the light sources or by switching on and off differing lights between image acquisition stages, in terms of practical application allowing potential for rapid or uncontrolled relative movement between the camera and the surface, this assumption is found to be most restrictive. In many industrial inspection applications it is often the case that the component surfaces tend to be moving rapidly. For example, in the case of a web inspection this could be in excess of $200 \mathrm{~m} / \mathrm{min}$. It is the ability to simultaneously acquire images under different lighting configurations that forms the basis of realising a dynamic photometric stereo (DPS) method.

\section{Towards Dynamic Photometric Stereo}

\subsection{Spectral Multiplexing}

It is useful to refer to each image / illumination configuration as a 'channel', where in order to minimise cross talk each channel must, as far as possible, be isolated from the others. Spectral multiplexing theoretically allows multiple images to be captured at a single point in space and time, where channel isolation may be realised in terms of light frequency separation. Two forms of spectral multiplexing present themselves, broad- and narrow-band.

Broadband colour photometric stereo. A number of related approaches have been described under what has been termed colour photometric stereo (CPS) or shape from colour, where colour channel separation is of the order of $100 \mathrm{~nm}$. As with much of the material relating to PS, CPS has generally been aimed at global shape recovery, rather than surface analysis per se. Nonetheless, CPS techniques are relevant to a discussion of the dynamic application of PS for surface analysis.

Drew [5] presents a method for recovering the shape of a Lambertian surface with unknown but uniform reflectance from a single composite RGB image. The method utilises a light that varies spectrally with direction and depends upon a linear 
relationship between the RGB sensor response and the surface normal direction. Effectively a colour Lambertian model is used, where albedo is replaced by a RGB vector giving the colour of the illuminate reflected by the surface. Drew et al. later extend the method to curved surfaces with uniformly coloured patches, however, the method described is limited to curved surfaces and will not work for flat planar patches. The assumption of uniform colour in their earlier work would seem to limit application. A somewhat similar approach is described by Yasushi [6] using only white matt objects. Barsky et al. [7] describe a method for classifying rough surfaces using colour and gradient information. Although colour images are used, the method reduces to the grey scale case and is not CPS in the sense described here. Tian et al. [8] similarly use a colour image to recover the shape of non-Lambertian surfaces. An extended light source with differing colour to that of the object is used so that specular and diffuse reflection components may be detected simultaneously. By using colour information, regions of dichromatic, termed hybrid in their paper, reflection are segmented from regions of diffuse reflection. Unfortunately, limitations are again placed on variation in object colour by assuming uniform colouring. Only monochromatic surfaces are considered. Christensen et al. [9] also describe a variation of the photometric stereo method, utilizing colour instead of grey scale images. Their colour photometric stereo method is really a refinement of grey scale photometric stereo, and has been used to recover the shape of a coloured object from two or more colour images under white illumination. However, when applied to a purely diffuse refection their method corresponds to grey scale photometric stereo of the same surface. As with the work of Barsky et al. this is effectively grey scale PS and not CPS.

It is useful to introduce a specific definition for CPS. This is necessary in order to distinguish it from those methods utilising multiple images and multiple lights of equal chromacity (where although colour data is captured, the method simply reduces to conventional grey scale PS), and other methods. Therefore the term CPS is used here to specifically refer to any method in which colour information is used in the recovery of surface shape or topography.

Methods that have generally utilised single RGB image acquisition and lighting that varies spectrally with direction are often limited to monochromatic surfaces. An interesting interpretation of the colour photometric stereo approach is described by Detlef et al. [10]. Here three illuminates, red, green and blue, two in a dark-field and one in a bright-field configuration, are used with a single colour line scan camera. The two dark-field illuminates are arranged in a symmetrical configuration about the camera axis. By measuring the difference between the two images, an estimation of surface slope or gradient is arrived at. However, as only two illuminates are used only one degree of surface orientation is recovered in this configuration. Hence, it is not possible to obtain a full bump map and this limits its potential for full 3D-feature description. As with other methods the approach would appear to be limited to monochromatic and also to nominally planar geometry.

Mahdavieh [11] identifies a general limitation of the CPS method. Assuming the RGB intensities at a given surface point are known, while the $\mathrm{x}, \mathrm{y}$ and $\mathrm{z}$ component of the surface normal and the RGB components of the surface reflectance spectrum remain unknown, the number of unknowns will exceed the number of equations that may be formulated. As such, the problem cannot be solved without introducing additional constraints. Mahdavieh proposes two solutions: either assume that the colour of 
the object is monochromatic and just as importantly known a priori or add an additional image, captured using a broad spectrum white light located close to the camera position. Unfortunately, because the white light will cause a coupling with the RGB lights it becomes necessary to also introduce some other form of multiplexing, i.e. spatial. An alternative approach is now introduced, termed narrow band PS it serves to alleviate those limitations associated with CPS.

Narrow band colour PS - a new approach. A number of key limitations have been shown to present themselves when attempting to use a broad band colour PS approach. Firstly, when deploying widely spaced channels of visible light, a coupling is found to exist between surface colour and surface gradient, in which it becomes impossible to determine whether an observed surface colour is due to an unknown arbitrary surface reflectance or whether it is due to unknown surface gradient. Secondly, in order to use a standard RGB colour camera, some 100nm must separate each colour channel. This means that a surface of fixed arbitrary colour will appear at differing intensities under each coloured illuminate (e.g. a red surface will exhibit low radiance under blue illumination and high radiance under red illumination). In previous work these problems have been overcome using a priori knowledge, for example, by assuming that the surface is monochromatic or by previously acquiring a separate colour image, later registered with the PS images. The alternative is to utilise narrow frequency channels that are closely spaced at around only $20 \mathrm{~nm}$ intervals or less. This new approach will be termed narrow channel or narrow band PS.

Figure 1 shows a typical spectral response for a red specimen surface with the region of closely spaced channels shown in the IR region of the spectrum. Approaching medium to long wave IR, i.e. 1.4 to 10 microns, further reduces sensitivity to changes in surface colour, i.e. different colours become metameric to one another. Also, it is known that both CCD and particularly CMOS cameras have excellent response in the IR region of the spectrum. Hence, using closely spaced channels within the infrared, surface colour data may be de-coupled from gradient data. In addition, if required an additional now de-coupled superimposed white channel (i.e. minus the IR) may be simultaneously included to provide fully registered colour data. This approach will be termed narrow infrared PS (NIRPS) [12]. A schematic configuration of the physical set-up is shown by Figure 2(a) with the detail of the camera optics shown in Figure 2(b).

The selection of appropriate filter optics becomes important in achieving good channel isolation and because of the limitations of real devices inevitably a compromise must be struck. For example, if the channel frequencies are spaced too widely then a given surface colour will appear with a different intensity within each channel causing variation in surface albedo to appear as topography in the bump map. If on the other hand the channels are too closely spaced then channel cross talk will be increased, distorting the recovered bump map by causing a shift in the observed position of the lights. In practice cross talk turns out to be less of an issue than albedo misinterpretation and can be reduced by calibration in which the contribution from the opposing two channels is subtracted from each channel. 


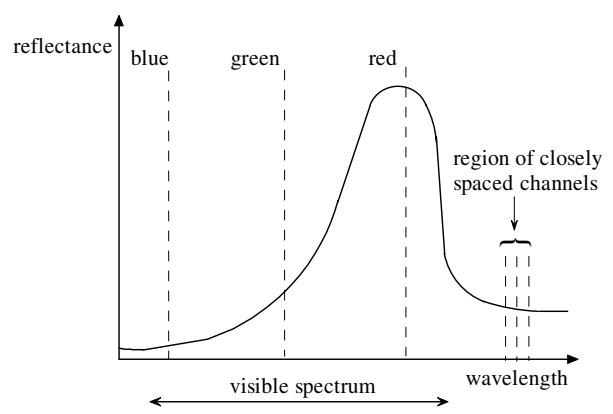

Fig. 1. Spectral distribution of a red surface
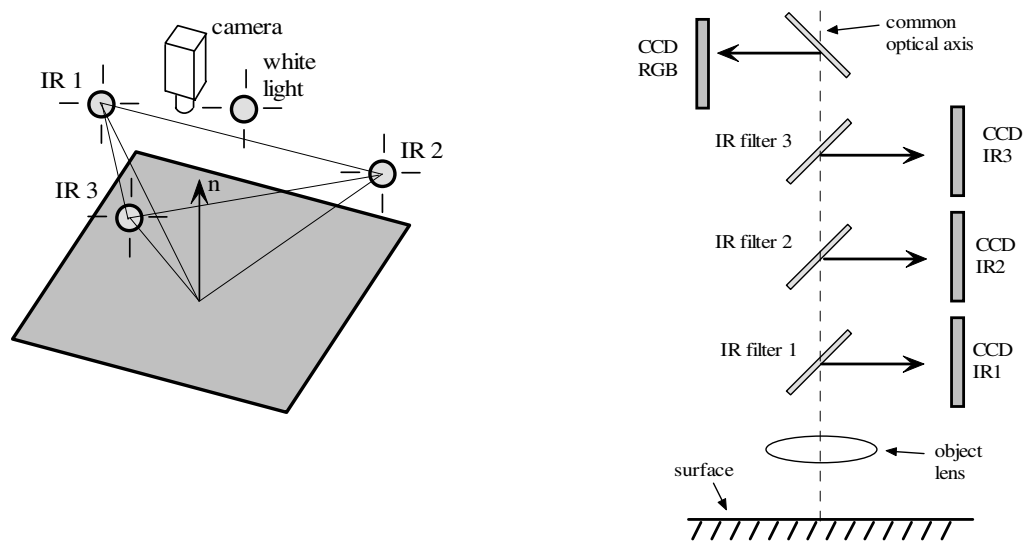

(a) Lighting and camera configuration

(b) Camera optics

Fig. 2. Narrow IR photometric stereo (NIRPS)

\subsection{Hybrid spatial / Frequency Multiplexing}

A limitation of the NIRPS technique is the inherent complexity of camera optics, as shown by Figure 2(b). This may be considerably reduced by adopting a hybrid approach. Here images are isolated both in terms of spectral frequency and also by a close spatial displacement of one or two lines of pixels. A schematic configuration is shown in Figure 3.

This allows for considerable simplification of camera and optics. Instead of using multiple CCDs arranged off a single optical axis, adjacent CCDs (as shown) or adjacent regions of a single CCD may be used. As with spectral multiplexing, the various illuminates may be flooded into the inspection area, simplifying illumination in comparison with spatial multiplexing techniques, while channel separation takes place at the camera. 


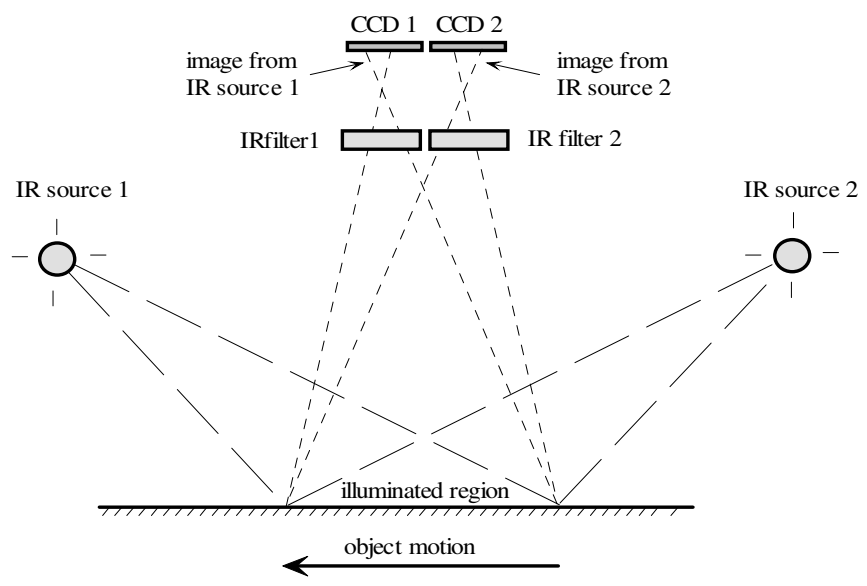

Fig. 3. Dynamic narrow infra-red photometric stereo (NIRPS)

\section{Experimental Work}

Static narrow IR photometric stereo (NIRPS). Initial static tests were performed using a ceramic surface exhibiting 3D topography with superimposed colouring. The surface includes a multi-coloured pattern and, at the top of the image, a threedimensional feature, which also has a colour that differs from the rest of the sample. Three differing IR sources, 700, 710 and 720nm were used to simultaneously illuminate the scene in a directional manner, while images were taken using matched camera filters, one for each illuminate. Narrow band-pass interference filters were used for channel separation.

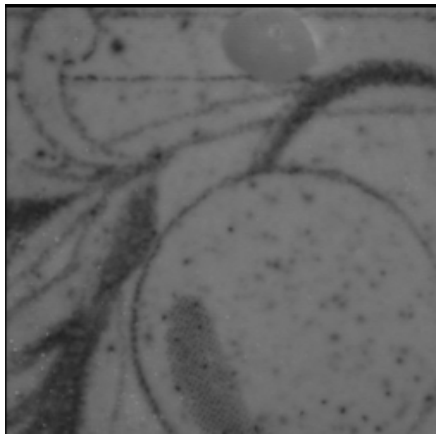

(a) Acquired image

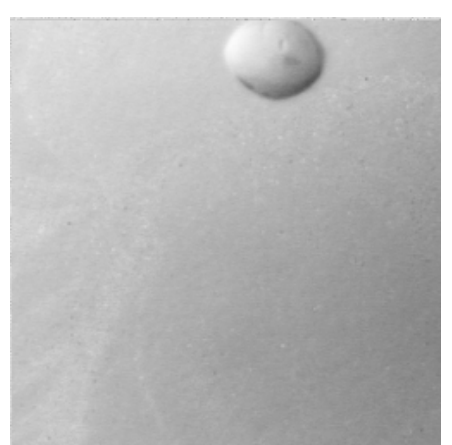

(b) Rendered bump map (topography)

Fig. 4. Narrow infra-red photometric stereo (NIRPS)

Figure 4 shows an albedo and a rendered bump map image. As can be seen, the surface topography has separated well in the rendered image, with only a faint rem- 
nant of the albedo remaining. Cross talk between channels, observed by measuring the response of say the $750 \mathrm{~nm}$ illuminate through the $730 \mathrm{~nm}$ filter was found to be negligible.

Dynamic application using line scan imaging. Figure 5 shows the results of applying dynamic NIRPS using simultaneous illumination from IR LED line lights and line scan camera acquisition. The surface was travelling at $30 \mathrm{~m} / \mathrm{minute}$.

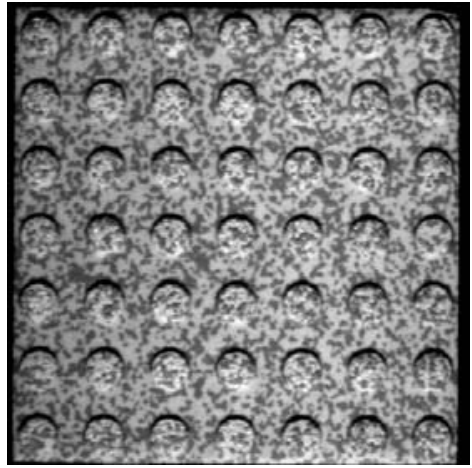

(a) Image $710 \mathrm{~nm}$ channel

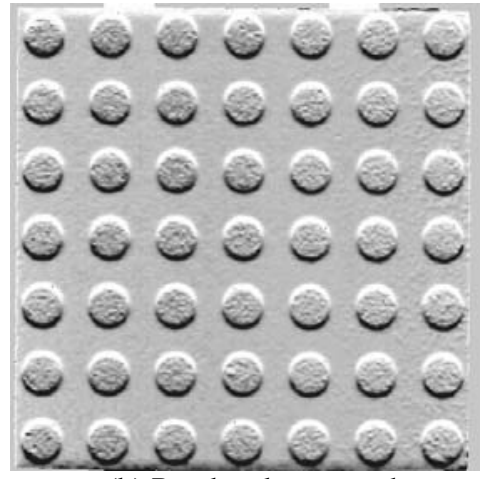

(b) Rendered topography

Fig. 5. Dynamic NIRPS operating at $30 \mathrm{~m} /$ minute

As can be seen, the albedo is generally very well removed.

\section{Conclusions}

A new technique termed dynamic photometric stereo (DPS) has been described. DPS represents an evolution in the theory of photometric stereo by extending application to moving surfaces, thereby for the first time allowing application to numerous industrial inspection tasks. The technique has been implemented using a method known as narrow infrared photometric stereo (NIRPS). Experimental results have shown that NIRPS may be used to isolate concomitant two- and three-dimensional features on fast moving objects.

\section{References}

1. Smith, M. L., Surface Inspection Techniques - Using the integration of innovative machine vision and graphical modelling techniques, Professional Engineering Publishing, ISBN 186058-292-3, (2000).

2. Smith, M. L., Smith, L. N., Machine vision inspection, in Xipend Xu (ed), Machining of Natural Stone, Trans Tech Publishing, ISBN 0-87849-927-X, (2003). 
3. Smith, M. L., Farooq, A. R., Smith, L. N., Midha, P. S., Surface texture inspecttion using conventional techniques applied to a photometrically acquired bump map, Sensor Review, Vol. 20, No. 4, (2000).

4. Smith, M. L., The analysis of surface texture using photometric stereo acquisition and gradient space domain mapping, Image and Vision Computing, Vol. 17, No. 14, (1999).

5. Drew, M. S., Shape from colour, Technical report, Centre for Systems Science/LCCR TR 92-07, School of Computing Science, Simon Fraser Uni, Vancouver, BC, (1992).

6. Yasushi, T., Shun'ichi, K., Tsunenori, H., A method for shape reconstruction of solid objects based on colour photometric stereo, IPSJ SIGNotes Computer Vision Abstract No. 080-16, (2001).

7. Barsky, S., Petrou, M., Classification of $3 \mathrm{D}$ rough surfaces using colour and gradient information recovered by colour photometric stereo, Proceedings of SPIE, 4553, pp. 10-19, (2001).

8. Tian, Y., Tsui, H. T., Extended light source model and reflectance map for nonLambertian surfaces, Journal of Optical Society of America, Vol. 14, No. 2, pp. 397-404, (1997).

9. Christensen, P.H. and Shapiro, L. G., Three-dimensional shape from color photometric stereo, International Journal of Computer Vision, Vol.13, No. 2, pp. 213-227, (1994).

10. Detlef, P., (inventor), Method and apparatus for automatic inspection of moving surfaces, European patent application EP 0898163 A1, (1999).

11. Mahdavieh, Y., Investigation of a colour photometric stereo vision system, $\mathrm{PhD}$ disertation, Faculty of Technology, University of Manchester, (1984).

12. Smith, M. L., and Smith, L. N., (inventors), 'Infra-red photometric stereo', patent application WO03/012412A2, filed July 2001. 